\title{
Integration of the Al-Tememe Transformation To find the Inverse of Transformation And Solving Some LODEs With (I.C)
}

\section{Ali Hassan Mohammed \\ University of Kufa \\ College of Education \\ of Women \\ Department of Mathematics}

Abass85@yahoo.com

Recived : $20 \backslash 4 \backslash 2015$

\section{Alaa Saleh Hadi \\ Al - Batul Secondary School for Girls in Wasit Governorate}

alaasalh.hadi@gmail.com

Revised : $14 \backslash 3 \backslash 2017$
Hassan Nadem Rasoul

University of Kufa

College of Computer

Science and Math

Department of Mathematics

Hassan.nadem.rasoul@gmail.com

Accepted : $14 \backslash 9 \backslash 2017$

\begin{abstract}
:
Our aim in this paper is to find the integration of Al-Tememe $(\mathcal{T})$ transformation to help us in finding $\left(\mathcal{T}^{-1}\right)$ for some functions without using of partition method and solve linear ordinary differential equations (LODEs) with variable coefficients by using (I.C) .
\end{abstract}

Mathematical subject classification:

\section{Introduction:}

We will use the new idea exists in [3] to find the integration of Al-Tememe transformation so it will give us the ability to find $\left(\mathcal{T}^{-1}\right)$ by new method for some functions.

\section{Basic definitions and concepts :}

\section{Definition 1: [1]}

Let $\mathrm{f}$ is defind function at a period $(a, b)$ then the integral transformation for $\mathrm{f}$ whose it's symbol $F(p)$ is defined as :

$$
F(p)=\int_{a}^{b} k(p, x) f(x) d x,
$$

Where $\mathrm{k}$ is a fixed function of two variables $\mathrm{x}$ and $\mathrm{p}$, called the kernel of the transformation, and $\mathrm{a}, \mathrm{b}$ are real numbers or $\mp \infty$, such that the integral above converges.

\section{Definition 2: [2]}

Al-Tememe transformation for the function $f(x) ; x>1$ is defined by the following integral :

$$
\mathcal{T}[f(x)]=\int_{1}^{\infty} x^{-p} f(x) d x=F(p)
$$

Such that this integral is convergent, $p$ is positive constant.

\section{Property 1: [2]}

Al-Tememe transformation is characterized by the linear property, that is:

$$
\mathcal{T}[A f(x)+B g(x)]=A \mathcal{T}[f(x)]+B \mathcal{T}[g(x)],
$$

Where $A, B$ are constants ,the functions $f(x)$, $g(x)$ are defined when $x>1$.

Al-Tememe transform of some fundamental functions are given in table(1) [2] :

From Al-Tememe definition and the above table, we get: 
Ali .H/Alaa .S/Hassan .N

\section{Theorem1:}

If $\mathcal{T}[f(x)]=F(p)$ and $\mathrm{a}$ is constant, then $\mathcal{T}\left[x^{-a} f(x)\right]=F(p+a)$.see [2]

\section{Definition 3: [2]}

Let $f(x)$ be a function where $(x>1)$ and $\mathcal{T}[f(x)]=F(p), f(x)$ is said to be an inverse for the Al-Tememe transformation and written as $\mathcal{T}^{-1}[F(p)]=f(x) \quad$, where $\mathcal{T}^{-1}$ returns the transformation to the original function.

\section{Property 2: [2]}

If $\quad \mathcal{T}^{-1}\left[F_{1}(p)\right]=f_{1}(x), \quad \mathcal{T}^{-1}\left[F_{2}(p)\right]=$ $f_{2}(x), \ldots, \mathcal{T}^{-1}\left[F_{n}(p)\right]=f_{n}(x)$ and $a_{1}, a_{2}, \ldots, a_{n}$ are constants then,

$$
\begin{aligned}
\mathcal{T}^{-1}\left[a_{1} F_{1}(p)+\right. & \left.a_{2} F_{2}(p)+\cdots+a_{n} F_{n}(p)\right] \\
& =a_{1} f_{1}(x)+a_{2} f_{2}(x)+\cdots \\
& +a_{n} f_{n}(x)
\end{aligned}
$$

Theorem 2: [2]

If the function $\mathrm{f}(\mathrm{x})$ is defined for $x>1$ and its derivatives $f^{(1)}(x), f^{(2)}(x), \ldots, f^{(n)}(x)$ are exist then:

$$
\begin{aligned}
\mathcal{T}\left[x^{n} f^{(n)}(x)\right]= & -f^{(n-1)}(1)-(p-n) f^{(n-2)}(1)-\cdots \\
& -(p-n)(p-(n-1)) \ldots(p \\
& -2) f(1)+(p-n) ! F(p)
\end{aligned}
$$

\section{Definition 4 : [4]}

A function $f(x)$ is piecewise continuous on an interval $[a, b]$ if the interval can be partitioned by a finite number of points $a=x_{0}<x_{1}<\cdots<x_{n}=$ $b$ such that:

1. $f(x)$ is continuous on each subinterval $\left(x_{i}, x_{i+1}\right)$, for $i=0,1,2, \ldots, n-1$

2. The function $\mathrm{f}$ has jump discontinuity at $\mathrm{x}_{\mathrm{i}}$, thus

$$
\begin{gathered}
\left|\lim _{x \rightarrow x_{i}} f(x)\right|<\infty, i=0,1,2, \ldots, n-1 ;\left|\lim _{x \rightarrow x_{i}} f(x)\right| \\
<\infty, \quad i=0,1,2, \ldots, n
\end{gathered}
$$

Note: A function is piecewise continuous on $[0, \infty)$ if it is piecewise continuous in $[0, A]$ for all $A>0$
Integration of the Laplace Transform [3]

If $f$ is piecewise continuous function on $[0, \infty]$ of exponential order $\alpha \geq 0$, and :

$$
\begin{gathered}
g(x)=\int_{0}^{x} f(u) d u \quad \Rightarrow \quad \mathcal{L}[g(x)]= \\
\frac{1}{p} \mathcal{L}[f(x)] ; \quad p \text { positive number. } \\
\Rightarrow g(x)=\int_{0}^{x} f(u) d u=\mathcal{L}^{-1}\left[\frac{F(p)}{p}\right]
\end{gathered}
$$

\section{Integration of Al-Tememe transforms:}

In ordinary differential equations it is also necessary

to compute Al-Tememe transform of an integral.

By the same method we can derive similar law to find a $\mathcal{T}^{-1}$ for some functions:

Consider,

$$
g(x)=\int_{1}^{x} f(u) d u \quad ; \quad g^{\prime}(x)=
$$

$f(x)$

To find $\mathcal{T}[g(x)]$

$$
\begin{aligned}
\because \mathcal{T}[g(x)] & =\int_{1}^{\infty} x^{-p} g(x) d x \\
\therefore \mathcal{T}[g(x)] & =\left.\frac{x^{-p+1}}{-p+1} \cdot g(x)\right|_{1} ^{\infty} \\
& \quad-\frac{1}{-p+1} \int_{1}^{\infty} x^{-p+1} \cdot f(x) d x
\end{aligned}
$$

$$
\begin{gathered}
=0+\frac{1}{p-1} \int_{1}^{\infty} x \cdot x^{-p} f(x) d x \\
\Rightarrow \mathcal{T}[g(x)]=\frac{1}{p-1} \mathcal{T}[x \cdot f(x)] ; p>1
\end{gathered}
$$

By take $\mathcal{T}^{-1}$ to both sides we get :

$$
g(x)=\mathcal{T}^{-1}\left[\frac{1}{p-1} \cdot \mathcal{T}[x \cdot f(x)]\right]
$$

$$
\therefore \mathcal{T}^{-1}\left[\frac{\mathcal{T}[x \cdot f(x)]}{p-1}\right]=\int_{1}^{x} f(u) d u=g(x) \mathbf{\square}
$$


Ali .H/Alaa .S/Hassan .N

Example 1: To find

$$
\mathcal{T}^{-1}\left[\frac{1}{(p-1)(p-5)}\right]
$$

We note that,

$$
\begin{aligned}
\mathcal{T}^{-1}\left[\frac{1}{(p-1)(p-5)}\right]=\mathcal{T}^{-1}\left[\frac{1 /(p-5)}{(p-1)}\right] \\
=\mathcal{T}^{-1}\left[\frac{\mathcal{T}\left(x^{4}\right)}{(p-1)}\right]=\mathcal{T}^{-1}\left[\frac{\mathcal{T}\left(x \cdot x^{3}\right)}{(p-1)}\right]
\end{aligned}
$$

Applying the previous relation we get :

$$
\begin{aligned}
\mathcal{T}^{-1}\left[\frac{\mathcal{T}\left(x \cdot x^{3}\right)}{(p-1)}\right] & =\int_{1}^{x} f(u) d u \\
& =\int_{1}^{x} u^{3} d u=\left.\frac{u^{4}}{4}\right|_{1} ^{x}=\frac{x^{4}}{4}-\frac{1}{4}
\end{aligned}
$$

Example 2: To find

$$
\mathcal{T}^{-1}\left[\frac{1}{(p-1)(p+2)(p-3)}\right]
$$

We note that,

$$
\begin{array}{r}
\mathcal{T}^{-1}\left[\frac{1}{(p-1)(p+2)(p-3)}\right] \\
=\mathcal{T}^{-1}\left[\frac{1 /(p+2)(p-3)}{(p-1)}\right] \\
\because \frac{1}{(p+2)(p-3)}=\frac{-1 / 5}{(p+2)}+\frac{1 / 5}{(p-3)} \\
\therefore \mathcal{T}^{-1}\left[\frac{1 /(p+2)(p-3)}{(p-1)}\right] \\
=\mathcal{T}^{-1}\left[\frac{\mathcal{T}\left(-1 / 5^{\left.x^{-3}+1 / 5^{x^{2}}\right)}\right.}{(p-1)}\right]
\end{array}
$$

Applying the previous relation we get :

$$
\begin{gathered}
=\mathcal{T}^{-1}\left[\frac{\mathcal{T}\left[x \cdot \left(-1 / 5^{\left.\left.x^{-4}+1 / 5^{x}\right)\right]}\right.\right.}{(p-1)}\right]=\int_{1}^{x} f(u) d u \\
=\int_{1}^{x}\left(-1 / 5^{u^{-4}+1 / 5} u\right) d u \\
=\left.\left(1 / 15^{-3}+1 / 10 u^{2}\right)\right|_{1} ^{x}=1 / 15^{x^{-3}+1 / 10} x^{2}-\frac{1}{6}
\end{gathered}
$$

Example 3: To find

$$
\mathcal{T}^{-1}\left[\frac{1}{(p-1)\left[(p-2)^{2}+4\right]}\right]
$$

We note that,

$$
\begin{aligned}
\mathcal{T}^{-1}\left[\frac{1 / 2 \cdot \frac{2}{(p-2)^{2}+4}}{(p-1)}\right] & =1 / 2 \mathcal{T}^{-1}\left[\frac{\frac{2}{(p-2)^{2}+4}}{(p-1)}\right] \\
= & 1 / 2 \mathcal{T}^{-1}\left[\frac{\mathcal{T}[x \cdot \sin (2 \ln x)]}{(p-1)}\right]
\end{aligned}
$$

Applying the previous relation we get :

$$
\begin{aligned}
& 1 / 2 \mathcal{T}^{-1}\left[\frac{\mathcal{T}[x \cdot \sin (2 \ln x)]}{(p-1)}\right]=1 / 2 \int_{1}^{x} f(u) d u \\
& =1 / 2 \int_{1}^{x} \sin (2 \ln u) d u
\end{aligned}
$$

$$
\begin{aligned}
& \int_{1}^{x} \sin (2 \ln u) d u \\
& =\left.u \sin (2 \ln u)\right|_{1} ^{x} \\
& -2 \int_{1}^{x} \cos (2 \ln u) d u
\end{aligned}
$$$$
=x \sin (2 \ln x)-0
$$$$
-2\left(\left.u \cos (2 \ln u)\right|_{1} ^{x}\right.
$$$$
\left.+2 \int_{1}^{x} \sin (2 \ln u) d u\right)
$$$$
=x \sin (2 \ln x)-2[x \cos (2 \ln x)-1]
$$$$
-4 \int_{1}^{x} \sin (2 \ln u) d u
$$$$
\Rightarrow \quad 5 \int_{1}^{x} \sin (2 \ln u) d u
$$

$$
=x \sin (2 \ln x)-2 x \cos (2 \ln x)
$$

$+2$

$\therefore \int_{1}^{x} \sin (2 \ln u) d u$

$=\frac{1}{5}[x \sin (2 \ln x)-2 x \cos (2 \ln x)$

$+2]$

so, $1 / 2 \int_{1}^{x} \sin (2 \ln u) d u$

$=\frac{1}{10}[x \sin (2 \ln x)$

$-2 x \cos (2 \ln x)+2]$

$\therefore \mathcal{T}^{-1}\left[\frac{1}{(p-1)\left[(p-2)^{2}+4\right]}\right]$

$=\frac{1}{10}[x \sin (2 \ln x)$

$-2 x \cos (2 \ln x)+2]$ 
Example 4: To find

$$
\mathcal{T}^{-1}\left[\frac{p+2}{(p+1)^{3}-8}\right]
$$

We note that,

$$
\begin{aligned}
\mathcal{T}^{-1}\left[\frac{p+2}{(p+1)^{3}-8}\right] & \mathcal{T}^{-1}\left[\frac{p+2}{[(p+1)-2]\left[(p+1)^{2}+2(p+1)+4\right]}\right] \\
= & \mathcal{T}^{-1}\left[\frac{(p+2)}{(p-1)\left(p^{2}+4 p+7\right)}\right] \\
= & \mathcal{T}^{-1}\left[\frac{p+2}{(p-1)\left[(p+2)^{2}+(\sqrt{3})^{2}\right]}\right] \\
& =\mathcal{T}^{-1}\left[\frac{p+2}{(p+2)^{2}+(\sqrt{3})^{2}}\right] \\
= & \int_{1}^{x} f(p-1) \\
& =\mathcal{T}^{-1}\left[\frac{\mathcal{T}\left[x^{-3} \cos (\sqrt{3} \ln x)\right]}{(p-1)}\right] \\
& \mathcal{T}^{-1}\left[\frac{\mathcal{T}\left[x \cdot x^{-4} \cos (\sqrt{3} \ln x)\right]}{(p-1)}\right] \\
& \\
& \\
&
\end{aligned}
$$

By applying the previous relation we get :

$$
\begin{gathered}
\mathcal{T}^{-1}\left[\frac{\mathcal{T}\left[x \cdot x^{-4} \cos (\sqrt{3} \ln x)\right]}{(p-1)}\right] \\
=\int_{1}^{x} u^{-4} \cos (\sqrt{3} \ln u) d u \\
=-1 /\left.3 u^{-3} \cos (\sqrt{3} \ln u)\right|_{1} ^{x} \\
-\frac{1}{\sqrt{3}} \int_{1}^{x} u^{-4} \sin (\sqrt{3} \ln u) d u \\
=-1 / 3 x^{-3} \cos (\sqrt{3} \ln x) \\
+1 / 3-\frac{1}{\sqrt{3}} \int_{1}^{x} u^{-4} \sin (\sqrt{3} \ln u) d u
\end{gathered}
$$

Ali .H/Alaa .S/Hassan .N$$
=-1 / 3 x^{-3} \cos (\sqrt{3} \ln x)+1 / 3
$$

$$
\begin{aligned}
& -\frac{1}{\sqrt{3}}\left[\left.\frac{-u^{-3}}{3} \sin (\sqrt{3} \ln u)\right|_{1} ^{x}\right. \\
& \left.+\frac{1}{\sqrt{3}} \int_{1}^{x} u^{-4} \cos (\sqrt{3} \ln u) d u\right] \\
& =-1 / 3 x^{-3} \cos (\sqrt{3} \ln x) \\
& +1 / 3+\frac{1}{3 \sqrt{3}} x^{-3} \sin (\sqrt{3} \ln x) \\
& -\frac{1}{3} \int_{1}^{x} u^{-4} \cos (\sqrt{3} \ln u) d u \\
& \Rightarrow \frac{4}{3} \int_{1}^{x} u^{-4} \cos (\sqrt{3} \ln u) d u \\
& =-1 / 3 x^{-3} \cos (\sqrt{3} \ln x) \\
& +1 / 3+\frac{1}{3 \sqrt{3}} x^{-3} \sin (\sqrt{3} \ln x) \\
& \int_{1}^{x} u^{-4} \cos (\sqrt{3} \ln u) d u \\
& =-\frac{1}{4} x^{-3} \cos (\sqrt{3} \ln x)+\frac{1}{4} \\
& +\frac{1}{4 \sqrt{3}} x^{-3} \sin (\sqrt{3} \ln x) \\
& =\frac{1}{4}\left[1+\frac{1}{\sqrt{3}} x^{-3} \sin (\sqrt{3} \ln x)\right. \\
& \left.-x^{-3} \cos (\sqrt{3} \ln x)\right]
\end{aligned}
$$

Example 5: To solve the differential equation:

$$
x y^{\prime}-2 y=\ln x ; y(1)=0
$$

We take Al-Tememe transformation to both sides of above by Th.2 and Table(1) :

$$
\begin{gathered}
-y(1)+(p-1) \mathcal{T}(y)-2 \mathcal{T}(y)=\frac{1}{(p-1)^{2}} \\
\mathcal{T}(y)=\frac{1}{(p-3)(p-1)^{2}}=\frac{1 /(p-3)(p-1)}{(p-1)} \\
=\frac{1 /\left[(p-2)^{2}-1\right]}{(p-1)}
\end{gathered}
$$


So,

$$
\mathcal{T}(y)=\frac{\mathcal{T}[x \cdot \sinh (\ln x)]}{(p-1)}
$$

After taking $\mathcal{T}^{-1}$ to both sides we get:

$$
\begin{gathered}
y=\mathcal{T}^{-1}\left[\frac{\mathcal{T}[x \cdot \sinh (\ln x)]}{(p-1)}\right] \\
=\int_{1}^{x} f(u) d u=\int_{1}^{x} \sinh (\ln u) d u \\
\because \sinh (\ln u)=\frac{e^{\ln u}-e^{-\ln u}}{2}=\frac{1}{2}\left(u-u^{-1}\right) \\
\therefore \int_{1}^{x} \sinh (\ln u) d u=\left.\frac{1}{2}\left(\frac{u^{2}}{2}-\ln u\right)\right|_{1} ^{x} \\
=1 / 4 x^{2}-1 / 2 \ln x-1 / 4 \\
\Rightarrow y=1 / 4 x^{2}-1 / 2 \ln x-1 / 4
\end{gathered}
$$

Example 6: To solve the differential equation:

$$
x^{2} y^{\prime \prime}-x y^{\prime}=x^{2} ; y^{\prime}(1)=0, y(1)=2
$$

We take Al-Tememe transformation to both sides of above :

$$
\begin{gathered}
\mathcal{T}\left(x^{2} y^{\prime \prime}\right)-\mathcal{T}\left(x y^{\prime}\right)=\mathcal{T}\left(x^{2}\right) \\
-y(1)-y^{\prime}(1)(p-2)+(p-2)(p-1) \mathcal{T}(y)+y(1) \\
-(p-1) \mathcal{T}(y)=\frac{1}{(p-3)} \\
(p-3)(p-1) \mathcal{T}(y)=\frac{1}{(p-3)} \\
\mathcal{T}(y)=\frac{1}{(p-1)(p-3)^{2}}=\frac{1 /(p-3)^{2}}{(p-1)}
\end{gathered}
$$

After taking $\mathcal{T}^{-1}$ to both sides we get:

$$
\begin{gathered}
y=\mathcal{T}^{-1}\left[\frac{1 /(p-3)^{2}}{(p-1)}\right]=\mathcal{T}^{-1}\left[\frac{\mathcal{T}(x \cdot x \ln x)}{(p-1)}\right] \\
=\int_{1}^{x} f(u) d u=\int_{1}^{x} u \ln u d u \\
\int_{1}^{x} u \ln u d u=\left.\left(\frac{1}{2} u^{2} \ln u-\frac{1}{4} u^{2}\right)\right|_{1} ^{x} \\
=\frac{1}{2} x^{2} \ln x-\frac{1}{4} x^{2}+\frac{1}{4} \\
\Rightarrow y=\frac{1}{2} x^{2} \ln x-\frac{1}{4} x^{2}+\frac{1}{4}
\end{gathered}
$$

\begin{tabular}{|c|l|l|r|}
\hline ID & \multicolumn{1}{|c|}{ Function, $\boldsymbol{f}(\boldsymbol{x})$} & \multicolumn{1}{|c|}{$\boldsymbol{F}(\boldsymbol{p})=\int_{\mathbf{1}}^{\infty} \boldsymbol{x}^{-\boldsymbol{p}} \boldsymbol{f}(\boldsymbol{x}) \boldsymbol{d} \boldsymbol{x}=\boldsymbol{T}[\boldsymbol{f}(\boldsymbol{x})]$} & $\begin{array}{c}\text { Regional of } \\
\text { convergence }\end{array}$ \\
\hline 1 & $k ; k=$ constant & $\frac{k}{p-1}$ & $\boldsymbol{p}>\mathbf{1}$ \\
\hline 2 & $x^{n}, \quad n \in R$ & $\frac{1}{p-(n+1)}$ & $\boldsymbol{p}>\boldsymbol{n}+\mathbf{1}$ \\
\hline 3 & $\ln x$ & $\frac{1}{(p-1)^{2}}$ & $\boldsymbol{p}>\mathbf{1}$ \\
\hline 4 & $x^{n} \ln x, n \in R$ & $\frac{1}{[p-(n+1)]^{2}}$ & $\boldsymbol{p}>\boldsymbol{n}+\mathbf{1}$ \\
\hline 5 & $\sin (a \ln x)$ & $\frac{a}{(p-1)^{2}+a^{2}}$ & $\boldsymbol{p}>\mathbf{1}$ \\
\hline 6 & $\cos (a \ln x)$ & $\frac{p-1}{(p-1)^{2}+a^{2}}$ & $\boldsymbol{p}>\mathbf{1}$ \\
\hline 7 & $\sinh (a \ln x)$ & $\frac{a}{(p-1)^{2}-a^{2}}$ & $\mid \boldsymbol{p}-\mathbf{1}>\boldsymbol{a}$ \\
\hline 8 & $\cosh (a \ln x)$ & $\frac{p-1}{(p-1)^{2}-a^{2}}$ & $|\boldsymbol{p}-\mathbf{1}|>\boldsymbol{a}$ \\
\hline
\end{tabular}

Table (1) [2]. 


\section{References :}

[1] Gabriel Nagy , " Ordinary Differential Equations " Mathematics Department, Michigan State

University ,East Lansing, MI, 48824.October 14, 2014.

[2] Mohammed, A.H. ,Athera Nema Kathem, " Solving Euler's Equation by Using New

Transformation", Karbala university magazine for completely [3] Paul Dawkins, "Differential
Equations http://tutorial.math.lamar.edu/terms.as pxm, 2007.

[4] William F. Trench , “ Elementary Differential Equations " Trinity University, 2013.
تكامل تحويل التميمي لايجاد معكوس التحويل وحل بعض المعادلات الخطية الاعتيادية الخاضعة لثروط ابتدائية
حسن ناظم رسول جامعة الكوفة
كلية علوم الحاسوب والرياضية

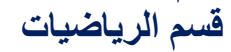
الاء صالح هادي ثانوية البتول للبنات$$
\text { في محافظة واسط }
$$
علي حسن محمد الكوفة جامعة الكوفة كلية التربية للبنات قسم الرياضيات

المستخلص :

هدفنا في هذا البحث هو ايجاد معكوس تحويل التميمي بأستخدام التكامل و ايجاد حل بعض المعادلات التهات التفاضلية

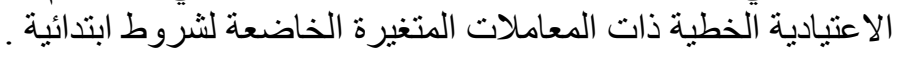

